\title{
Instructions pour les auteurs
}

Animal Genetic Resources/Resources génétiques animales/ Recursos genéticos animales est un journal trilingue, publié trois fois par an en ligne (http://journals.cambridge.org/AGR) et en version imprimée. Les articles principaux sont publiés en anglais, français ou espagnol avec un résumé dans les trois langues. Le journal est publié depuis 1983 et tous les anciens numéros sont disponibles à l'adresse électronique http://dad. fao.org/cgi-bin/EfabisWeb.cgi?sid=-1,refcat_50000044.

On encourage la présentation d'articles provenant du monde entier à publier dans le journal. Les auteurs qui ne parlent aucune des trois langues admises sont encouragés à chercher de l'aide à cet égard avant de présenter leurs manuscrits.

\section{Déclaration de mission}

Le journal fait office de forum international pour la publication d'articles concernant la gestion des ressources zoogénétiques pour l'alimentation et l'agriculture. Il aborde en particulier les thèmes suivants: la caractérisation phénotypique et moléculaire; les enquêtes et le suivi; la mise en valeur (amélioration génétique); l'utilisation durable; la conservation; le renforcement des capacités au sein des communautés d'éleveurs et de pasteurs; et les politiques et les institutions.

Les éditeurs accueillent favorablement tous les articles abordant les thèmes indiqués ci-dessus. Un intérêt particulier sera attribué aux articles concernant les races et les technologies en faveur de la gestion durable des systèmes de production extensive ou semi-intensive dans le monde, qui représentent la plus grande partie des terres consacrées à l'élevage et une partie considérable de la production provenant de l'élevage.

Le journal soutient la mise en œuvre du Plan d'action mondial pour les ressources zoogénétiques, le cadre internationalement convenu en matière de gestion des ressources animales et la Convention sur la diversité biologique.

\section{Déni de responsabilité}

Les opinions exprimées dans les articles publiés dans Animal Genetic Resources/Resources génétiques animales/Recursos genéticos animales sont celles du/des auteur(s) et ne reflètent pas nécessairement les politiques de la FAO ou les opinions des éditeurs ou des institutions pour lesquelles ils travaillent.

\section{Révision par les pairs}

Deux experts s'occuperont de la révision complète des manuscrits présentés pour la publication dans Animal Genetic Resources/Resources génétiques animales/Recursos genéticos animales. L'opportunité ou non de publier un manuscrit sera jugée par les réviseurs et par les éditeurs, et la décision finale sur l'article appartient aux éditeurs.

\section{Types d'articles}

Articles de recherche - Seront prises en considération pour leur publication sur AGR les études sur la gestion des ressources animales. On encourage les auteurs à envoyer des photographies de haute qualité avec les manuscrits. S'il s'agit de photographies d'animaux, il faudra montrer les races en question dans leur milieu naturel de production.

Révisions - Occasionnellement, des articles contenant une révision aux niveaux national, régional ou mondial des développements d'un ou de plusieurs aspects se rapportant à la gestion des ressources animales seront pris en considération. Ces articles pourront inclure les mises à jour des différents domaines de la gestion des ressources animales.

Articles spécifiques - Ponctuellement, des articles sur des thèmes spécifiques pourront être demandés pour la publication lorsque les éditeurs le jugeront nécessaire.

Autre matériel pour publication - On encourage les lecteurs à envoyer par courrier électronique à l'adresse AnGR-Journal@fao.org:

- la révision ou la proposition de livres

- les conclusions et les recommandations résultant de réunions, d'ateliers et de conférences importants

- les informations sur des cours de formation et sur les principaux événements régionaux, nationaux et internationaux.

\section{Originalité et droits d'auteur}

Pour que le manuscrit soit considéré pour la publication dans le journal, il faut qu'il n'ait pas été publié auparavant, ni qu'il soit en cours de révision pour la publication dans d'autres ouvrages. (Les chiffres publiés auparavant peuvent s'utiliser avec parcimonie dans les révisions, à condition d'en avoir obtenu l'autorisation.) Avant la publication, il faut que l'auteur principal du manuscrit signe et renvoie aux éditeurs le formulaire d'autorisation et d'accord de transfert des droits d'auteur (les auteurs principaux signent au nom de tous les co-auteurs). Le formulaire sera envoyé à l'auteur principal avec l'épreuve de l'article pour la publication.

\section{Paternité}

Les articles écrits par plusieurs auteurs sont révisés en présumant que tous les auteurs ont matériellement participé à la recherche signalée, ont approuvé le manuscrit présenté et approuvent sa présentation. Leur contribution comprend la conception et la création du projet, la performance d'expériences et/ ou l'analyse et l'interprétation des données. Les auteurs devront avoir apporté une contribution intellectuelle considérable à la rédaction et à la révision critique du manuscrit. 


\section{Présentation du manuscrit}

Tous les manuscrits seront présentés en ligne à l'adresse électronique http://journals.cambridge.org/AGR. Aucune charge par page ne sera requise de l'auteur.

On accusera réception du manuscrit, on attribuera un numéro de référence et le manuscrit sera envoyé pour révision. Vous devrez toujours indiquer le numéro de référence de votre manuscrit dans toute correspondance ultérieure.

Il faut suivre avec attention les instructions ci-après (pour de plus amples détails, voir Préparation et style du manuscrit).

- Les manuscrits se présenteront en anglais, français ou espagnol. Si votre manuscrit est en français ou en espagnol, il faudra ajouter un résumé et les mots clés dans cette langue ainsi qu'en anglais. On ajoutera à tous les articles publiés un résumé en anglais, français et espagnol. On appréciera si, dans la mesure du possible, les auteurs fournissent un résumé dans les trois langues, car les services de traduction seraient ainsi réduits et le traitement du manuscrit serait par conséquent plus rapide.

- Le format de fichier préféré pour la présentation est Microsoft Word. Word Perfect ou d'autres fichiers de traitement de texte ne sont pas acceptés. Les tableaux seront inclus au même fichier, mais à la fin du document. Les paramètres fictifs seront utilisés dans le texte pour indiquer leur positionnement.

- Les figures se présenteront en tant que fichiers séparés et en résolution publiable (pour de plus amples détails voir Préparation et style du manuscrit).

- Une lettre d'envoi sera envoyée dans un fichier séparé. La lettre signalera la catégorie d'appartenance du manuscrit (voir annexe 1) et fournira des informations sur l'auteur principal (numéro de téléphone, de télécopieur et adresse électronique).

- Les fichiers indiqueront le nom de l'auteur principal de l'article, soit en entier soit abrégé.

- Les copies imprimées du manuscrit, des tableaux et des figures ne sont pas requises et ne devront pas être envoyées.

Veuillez noter que la correspondance relative aux manuscrits présentés et révisés se fera uniquement avec l'auteur principal.

\section{Préparation et style du manuscrit}

Les manuscrits se présenteront à «double» interligne. Toutes les pages seront numérotées à commencer de la page du titre. Les marges seront d'au moins $2,5 \mathrm{~cm}$ pour tous les côtés. La police de caractère sera Arial.

Auteurs et institutions pour lesquelles ils travaillent - Les noms des auteurs et les institutions pour lesquelles ils travaillent se présenteront comme indiqué ci-après:

E.C. Quispe ${ }^{1}$, T.C. Rodríguez ${ }^{2}$, L.R. Iñiguez ${ }^{3}$ et J.P. Mueller ${ }^{4}$

${ }^{1}$ Universidad Nacional de Huancavelica, Huancavelica, Perú; ${ }^{2}$ Universidad Mayor de San Andrés, La Paz, Bolivia; ${ }^{3}$ Cochabamba, Bolivia; ${ }^{4}$ Instituto Nacional de Tecnología Agropecuaria, Bariloche, Argentina

Correspondance à envoyer à: E.C. Quispe, Universidad Nacional de Huancavelica, Huancavelica, Perú. Adresse électronique: edgarquispe62@yahoo.com
Sur la première page du manuscrit, on indiquera le titre de l'article qui ne devra pas dépasser les 50 caractères.

Le résumé ne sera pas structuré (c'est-à-dire, sans sous-titres), mais devra fournir au lecteur une brève description de l'article. Il inclura une introduction succincte à l'article, la méthode utilisée, les résultats principaux et les conclusions. Le résumé ne dépassera pas les 210 mots en anglais et les 250 mots en français et en espagnol. Une liste de mots clés ou de termes (entre trois et cinq) pour le sommaire suivra le résumé et les mots-clés seront séparés par des virgules. Le résumé et les mots-clés se présenteront dans la même langue du manuscrit ainsi qu'en anglais.

Le corps $\boldsymbol{d}$ u manuscrit commencera à la page 3 et une nouvelle page sera utilisée pour les références. Les lignes du texte seront numérotées, le manuscrit sera structuré et tous les titres et les sous-titres seront numérotés (par exemple, 1, 1.1, 1.1.1, etc.). Il est toutefois important d'éviter les références croisées avec ces numéros car le bureau d'édition enlèvera la numérotation et appliquera des styles de titre dans la version finale.

Les articles de recherche devront en outre inclure les titres suivants: Matériels et méthodes; Résultats; Débat; Conclusions.

La longueur maximale du corps du manuscrit ne dépassera pas les 10 pages du journal (environ 8500 mots). Les communications brèves ne dépasseront pas 1 page (environ 750 mots ou, s'il y a également une image, 550 mots).

Les tableaux seront tous numérotés en suivant l'ordre d'apparition dans le texte (tableau 1, 2 etc.). Chaque tableau sera sur une page séparée (à la fin du document) avec le numéro et le titre au-dessus du tableau et d'éventuelles notes au-dessous.

Les figures seront toutes numérotées en suivant l'ordre d'apparition dans le texte (figure 1, 2 etc.). Il faudra écrire les lettres des parties a, b, c, etc. en italique et prévoir des légendes pour chaque figure. Les figures se présenteront, si possible, dans un fichier TIFF ou EPS, de préférence dans la taille approximative à utiliser pour la reproduction. Les illustrations graphiques seront fournies en noir et blanc avec une résolution de 1200 ppp; les artwork combinaisons (ligne/ton) avec un résolution de 800 ppp; les illustrations en demi-ton noir et blanc seront sauvegardées en mode «niveau de gris» avec une résolution de 300 ppp; les illustrations en demi-teinte de couleurs seront enregistrées en mode CMJN avec une résolution de 400 ppp. Il faudra obtenir toutes les autorisations nécessaires.

Abréviations et unités SI - L'utilisation des abréviations, à part celles qui sont largement employées, est vivement déconseillée. Elles ne seront utilisées que si elles améliorent la compréhension du manuscrit. Les sigles s'écriront en entier la première fois qu'elles sont employées. Il faudra utiliser les unités du système métrique $(\mathrm{SI})$.

\section{Remerciements}

Dans cette section, les auteurs remercieront pour tout appui reçu des institutions et d'autres sources de soutien pour le travail inscrit dans leur article. On peut ajouter également dans cette section la contribution d'autres particuliers ayant aidé dans le travail de recherche, mais n'étant pas inclus en tant qu'auteurs. 
Les remerciements seront placés après le corps principal du texte avant les références. En cas d'absence de remerciements, le titre sera toutefois écrit et suivi par l'indication «aucun remerciement».

\section{Déclaration d'intérêts}

On est en présence d'un conflit d'intérêts lorsqu'un auteur a des intérêts qui pourraient influencer de façon inappropriée son jugement, même si ce jugement n'est pas en fait influencé. Pour cette raison, les auteurs doivent révéler les conflits d'intérêts potentiels pour que d'autres puissent juger de ces effets. Au moment de la présentation du manuscrit, les auteurs révéleront tout arrangement ou rapport financier pertinent avec le manuscrit présenté et qui pourrait être perçu comme pouvant porter un préjudice potentiel à l'article. Les auteurs révéleront également les intérêts non financiers qui pourraient être pertinents dans ce contexte. Il faudra également déclarer l'absence d'intérêts pertinents. Cette obligation s'applique à tous les auteurs d'un article et à toutes les catégories d'articles.

\section{Références}

Toute référence présente dans le texte devra apparaître sur la liste des références, et chaque entrée de la liste aura été citée au moins une fois dans le texte. Les références iront en ordre alphabétique du nom de l'auteur, suivi de l'année.

Exemples:

\section{Référence sur une revue:}

Köhler-Rollefson, I. 1992. The camel breeds of India in social and historical perspective. Animal Genetic Resources Information 10: 53-64.

2 Lorsqu'il s'agit de plus d'un auteur:

Matos, C.A.P., Thomas, D.L., Gianola, D., Tempelman, R. J. et Young, L.D. 1997. Genetic analysis of discrete reproductive traits in sheep using linear and non-linear models: 1. Estimation of genetic parameters, Journal of Animal Science 75: 76-87.

3 Dans le cas d'un livre ou d'une publication ad hoc, par exemple, un rapport, une thèse:

FAO, 2007. Plan mondial d'action pour les ressources zoogénétiques et la Déclaration d'Interlaken. Organisation des Nations Unies pour l'alimentation et l'agriculture, Rome, Italie (disponible à l'adresse électronique http:// www.fao.org/docrep/010/a1404f/a1404f00.htm).

van der Werf, J., Graser, H-U., Frankham, R. et Gondro, C. (éds.) 2009. Adaptation and fitness in animal populations. Evolutionary and breeding perspectives on genetic resources management. Springer.

4 S'il s'agit d'un acte d'une réunion:

Abad, M., Arrigo, J., Gibbons, A., Lanari, M.R., Morris, G. et Taddeo, H. 2002. Breeding scheme for Angora goat production in North Patagonia. Actes du Septième congrès mondial sur l'application de la génétique à l'élevage, 19-23 août 2002, Montpellier, France, 12-14.

\section{Dans le cas d'informations hébergées sur un site Web:}

FAO. 2010. Domestic Animal Diversity Information System, http://www.fao.org/dad-is/, Organisation des Nations Unies pour l'alimentation et l'agriculture, Rome, Italie.

Dans le cas d'un travail ayant été accepté pour la publication, mais n'ayant pas encore été publié, on écrira «sous presse» à la place de l'année de la publication. Il ne faudra pas écrire l'année prévue de la publication.

\section{Documentation supplémentaire en ligne}

La plate-forme en ligne donne la possibilité aux auteurs d'ajouter des données qui seraient autrement impossibles ou pas pratiques à inclure à la version imprimée. Les auteurs pourront inclure des tableaux et des figures ainsi que des données comme des vidéos, des images/structures en trois dimensions, des ensembles de données très détaillées et d'autres matériels supplémentaires ne convenant pas à la reproduction sur papier. Tout le matériel supplémentaire se présentera avec le manuscrit original. Les données supplémentaires seront indiquées dans le texte par le préfixe «S» (par exemple, tableau supplémentaire $\mathrm{S} 1$, figure supplémentaire $\mathrm{S} 1$ ). Les fichiers supplémentaires ne seront pas révisés et seront publiés tels que reçus. Les éditeurs devront approuver la publication électronique de ce matériel. Le manuscrit devra être autonome et se suffire à lui-même, sans le matériel supplémentaire (dans l'intérêt des lecteurs ayant uniquement accès à la copie papier).

\section{Processus d'examen}

Les manuscrits présentés au journal seront examinés par deux réviseurs externes et évalués par un des éditeurs. Si les éditeurs considèrent que l'article n'est pas pertinent avec ce journal ou que l'examen ne sera pas favorable, l'article pourra être renvoyé à l'auteur après l'examen initial des éditeurs. Ce processus de refus rapide permet à l'auteur de présenter immédiatement son travail ailleurs pour publication. Les manuscrits seront également refusés par les éditeurs s’ils ne sont pas conformes aux recommandations prévues pour leur préparation. Tous les efforts seront faits pour communiquer aux auteurs la décision de l'examen dans un délai de six semaines après la réception du manuscrit. Si les éditeurs demandent des révisions au manuscrit avant sa publication, on accordera un délai maximum d'un mois pour ces révisions.

\section{Epreuves}

L'éditeur se réserve le droit de réviser les manuscrits pour veiller à ce que la grammaire et l'orthographe soient cohérentes avec le style du journal. L'auteur principal recevra les épreuves en page pour la correction. Ces épreuves seront contrôlées et renvoyées dans un délai de deux jours après la réception. L'éditeur se réserve le droit de charger les auteurs en cas de correction excessive d'erreurs non typographiques. 